\title{
APLICACIÓN DE BORO EN EUCALIPTO: COMPARACIÓN DE FUENTES ${ }^{1}$
}

\author{
Marcelo Gabriel Ferrando² e José Pedro Zamalvide ${ }^{2}$
}

\begin{abstract}
RESUMEN - La deficiencia de boro (B) en sistemas forestales ha sido reportada en diferentes especies de pino y eucalipto, verificándose importantes mejoras en la producción y/o calidad de madera, con el agregado de este nutriente. La baja retranslocación del B dentro de la planta hace necesario un aporte constante para satisfacer las demandas del cultivo. Al ser un nutriente muy poco retenido por el suelo está sujeto a pérdidas por lixiviación. El uso de fertilizantes solubles brinda una solución a corto plazo, muy dependiente de situaciones ambientales, mientras que las fuentes de liberación lenta permitirían un aporte más constante y por más tiempo. El objetivo de este trabajo fue evaluar la disponibilidad en el tiempo del B proveniente de diferentes fuentes, a través de cambios en las concentraciones foliares de Eucalyptus globulus (Labille) y Eucalyptus grandis (Hill ex Maiden), en distintas situaciones de suelos y manejo. Se instalaron tres experimentos de campo de comparación de fuentes boratadas (borato de sodio vs ulexita), aplicadas en cobertura, a árboles con seis meses de transplantados, en distintos sitios experimentales de Uruguay. A los 6, 12 y 24 meses luego de la fertilización se evaluaron las concentraciones foliares de B. La ulexita mostró una alta solubilidad y baja residualidad, con similar eficiencia que el borato de sodio como aporte de B para los eucaliptos. La dosis de B aplicada ( $4 \mathrm{~g}$ de $\mathrm{B}$ por planta) parecería ser suficiente para alcanzar niveles foliares que podrían considerarse de suficiencia, sin llegar a niveles de toxicidad.
\end{abstract}

Keywords: Eucalyptus, Solubor and Ulexita.

\section{APLICAÇÃO DE BORO EM EUCALIPTO: COMPARAÇÃO DE FONTES}

\begin{abstract}
RESUMO - A deficiência de boro (B) em sistemas de produção florestal tem sido relatada em várias espécies de pinheiros e eucaliptos, verificando-se melhorias significativas na produção elou qualidade da madeira, com a adição desse nutriente. A baixa translocação de B dentro da planta faz necessário um fornecimento constante para atender as demandas da cultura. Sendo um nutriente pouco retido pelo solo, fica sujeito a perdas por lixiviação. O uso de adubos solúveis fornece uma solução de curto prazo, altamente dependente das condições ambientais, entretanto, as fontes de liberação lenta permitiriam um fornecimento mais constante e por mais tempo. O objetivo deste estudo foi avaliar a disponibilidade de B no tempo a partir de duas fontes, através de mudanças nas concentrações de folha de Eucalyptus globulus (Labill) e Eucalyptus grandis (Hill ex Maiden), em diferentes solos e situações de gestão. Foram instalados três experimentos de campo comparando fontes de B (ulexita vs borato de sódio), aplicadas em cobertura, em árvores com seis meses de transplante, em diferentes locais experimentais do Uruguai. Em 6, 12 e 24 meses após a adubação, foram avaliadas as concentrações foliares de B. A ulexita mostrou alta solubilidade e baixo teor residual, com eficiência similar à de borato de sódio na contribuição de B para o eucalipto. A dose de B aplicada (4 g de B por árvore) parece ser adequada para alcançar níveis considerados de suficiência foliar sem atingir níveis tóxicos.
\end{abstract}

Palavras-chave: Eucalyptus, Solubor e Ulexita.

\footnotetext{
${ }^{1}$ Recebido em 09.03.2012 aceito para publicação em 04.06.2012

${ }^{2}$ Facultad de Agronomía, Universidad de la República Uruguai. E-mail: < marceloferrando2@ gmail.com >e < zamal@st.com.uy>.
} 


\section{INTRODUCCIÓN}

La forestación en Uruguay se realiza mayoritariamente en suelos de baja fertilidad, en los cuales las plantas son generalmente fertilizadas solo con nitrógeno y fósforo. Sin embargo, en algunos casos, se han observado deficiencias de boro (B), especialmente en suelos desarrollados sobre Basamento Cristalino, Areniscas Cretácicas y Areniscas de Tacuarembó.

La deficiencia de B es más común que la deficiencia de cualquier otro micronutriente, $\mathrm{y}$ ha sido reportada en 132 cultivos comerciales en más de 80 países (SHORROCKS, 1997). Diversos autores (VAIL et al., 1961; STONE; WILL, 1965; COOLING; JONES, 1970; HOPMANS; FLINN, 1984; DELL, 1996), detectaron síntomas de deficiencia de B en diferentes especies de pino y eucalipto, verificando importantes mejoras en la producción y/o calidad luego de aplicar este nutriente al suelo o a la planta.

Los síntomas de deficiencia de B en eucaliptos han sido ampliamente descritos (SAVORY, 1962; TOKESHI et al., 1976; DELL; MALAJCZUK, 1994; MALAVOLTA et al, 1997; SILVEIRA et al., 1998; LEITE et al., 2010) incluyendo, entre otros: deformaciones en puntos de crecimiento, hojas nuevas pequeñas, deformes y con clorosis, acortamiento de los entrenudos. En situaciones de deficiencia severa se produce la muerte o secado apical ("dieback"), con la consiguiente pérdida de la dominancia y el rebrote de las yemas laterales (SILVEIRA et al., 2002). El secado apical ocurre generalmente el primer año luego del transplante, repercutiendo en el crecimiento al segundo y tercer año. Si en este período la deficiencia no fue muy severa, la planta puede recuperarse (SAVORY, 1962), posiblemente debido a una mayor exploración radicular luego de que la planta adquiere un cierto tamaño.

La falta de B disminuye también la viabilidad y vigor del polen (DUGGER, 1983; MATOH, 1997) y/o de la semilla (DELL; HUANG, 1997), siendo fundamental para la cicatrización de heridas o rajaduras de la corteza, por lo que su deficiencia favorecerá la infección por hongos (MALAVOLTA et al., 1997; SILVEIRA et al., 1998), algunos de los cuales pueden causar importantes pudriciones en el duramen, con la consecuente debilidad estructural y aumento de quebraduras por viento (REALI, 2000). La adaptación a las situaciones de deficiencia de B será diferente dependiendo del clon utilizado (MATTIELLO et al., 2009).
El B es considerado inmóvil a través del floema para la mayoría de las plantas (incluidos los eucaliptos), aunque existen algunos reportes en los cuales muestra mayor movilidad, asociado a la producción de cantidades significativas de polioles (LEITE et al., 2008; BROWN; SHELP, 1997). Cuando se comporta como inmóvil, al no poder ser retranslocado, se requiere un aporte constante que satisfaga las demandas en cada momento (MENGEL; KIRKBY, 2001). Dado que la forma predominante de $\mathrm{B}$ en la solución (en suelos con $\mathrm{pH}$ entre 5 y 9) es la de ácido bórico no disociado $\left(\mathrm{H}_{3} \mathrm{BO}_{3}{ }^{0}\right)$, altamente soluble, en suelos profundos y bien drenados, luego de períodos de abundantes lluvias, el B de la solución puede ser lixiviado en profundidad (HAVLIN et al., 2005), generándose posibles momentos de deficiencias.

Las principales fuentes de B utilizadas en Uruguay son fuentes solubles como el ácido bórico $\left(\mathrm{H}_{3} \mathrm{BO}_{3}\right.$; $17,5 \%$ de B), bórax $\left(\mathrm{Na}_{2} \mathrm{~B}_{4} \mathrm{O}_{7} \cdot 10 \mathrm{H}_{2} \mathrm{O} ; 11,3 \%\right.$ de $\left.\mathrm{B}\right)$ y Solubor ${ }^{\circledR}\left(\mathrm{Na}_{2} \mathrm{~B}_{8} \mathrm{O}_{13} \cdot 4 \mathrm{H}_{2} \mathrm{O} ; 20,8 \%\right.$ de B $)$. Recientemente han comenzado a utilizarse fuentes menos solubles originadas de roca molidas con boratos de sodio y calcio (tipo ulexita, $\mathrm{NaCaB}_{5} \mathrm{O}_{9} \cdot 8 \mathrm{H}_{2} \mathrm{O}$ ), sin tratamientos químicos (tras la extracción hay un proceso de secado, molido y concentrado) y, por lo tanto, de menor costo por unidad de B que las fuentes solubles, con concentraciones que oscilan entre $10 \%$ y $17 \%$ de B.

El uso de fuentes solubles brinda una solución a corto plazo y muy dependiente de situaciones ambientales. Períodos con abundantes lluvias pueden ocasionar pérdidas por lixiviación del B aplicado, arrastrándolo hasta zonas alejadas de las raíces. El uso de fuentes de liberación lenta puede minimizar este efecto, puesto que estas fuentes reponen gradualmente el B perdido por lixiviación o absorbido por las plantas, lo que no implica que igualmente pueda producirse alguna deficiencia temporal.

Existen pocos reportes de estudios comparativos de aplicaciones minerales a campo, siendo la mayoría de éstos en ensayos maceteros y con el objetivo de cuantificar toxicidad y lixiviación, pero no la respuesta de los cultivos. Anivel nacional existen escasas referencias de estudios con B (MÉNDEZ, 2003; SAYAGUÉS; MÉNDEZ, 2006), todos con fuentes solubles. Según Shorrocks (1997), los resultados de estudios de eficiencia de aplicación de $\mathrm{B}$ usando diferentes minerales triturados

Revista Árvore, Viçosa-MG, v.36, n.6, p.1191-1197, 2012 
no serían comparables. Mientras que en Nueva Zelanda se utiliza ulexita como un borato de acción lenta, en Brasil es considerada como semejante al bórax.

El objetivo de este trabajo fue evaluar los cambios de disponibilidad en el tiempo del B proveniente de dos diferentes fuentes (una soluble y otra parcialmente soluble en agua), a través de los cambios en las concentraciones foliares de Eucalyptus globulus (Labille) y Eucalyptus grandis (Hill ex Maiden), en distintas situaciones de suelos y manejo.

\section{MATERIALES Y MÉTODOS}

En otoño de 2005 se instalaron 3 experimentos de campo para la comparación de fuentes de B, en diferentes sitios del Uruguay, sobre suelos que por sus características hacen posible la aparición de deficiencias de este nutriente (Basamento Cristalino, Areniscas Cretácicas y Areniscas de Tacuarembó). En los Cuadros 1 y 2 se muestra la ubicación y características de los diferentes sitios experimentales.
Los sitios 1 y 2 fueron forestados con Eucalyptus grandis (Hill ex Maiden), y el sitio 3 con Eucalyptus globulus (Labille).

Se utilizaron dos fertilizantes boratados: una fuente soluble en agua, del tipo borato de sodio $\left(\mathrm{Na}_{2} \mathrm{~B}_{8} \mathrm{O}_{13} \cdot 4 \mathrm{H}_{2} \mathrm{O}\right)$, con $20,8 \%$ B (Solubor $\AA$ ) y otra parcialmente soluble en agua, del tipo ulexita o boronatrocalcita $\left(\mathrm{NaCaB}_{5} \mathrm{O}_{9} \cdot 8 \mathrm{H}_{2} \mathrm{O}\right)$, con $17,6 \% \mathrm{~B}$ (LBF50), granulado (malla 2-4 mm). La dosis utilizada, tanto a la instalación como en la refertilización, fue de $4 \mathrm{~g}$ de B por planta. Al momento de la instalación de los experimentos, los árboles tenían seis meses de transplantados, realizándose la refertilización (en los tratamientos que correspondía) al año de la instalación del experimento (18 meses desde el transplante). La aplicación del fertilizante se realizó en cobertura, distribuida a ambos lados del árbol, a una distancia aproximada de $30 \mathrm{~cm}$. Excepto por la fertilización boratada, el resto de las prácticas agrícolas fueron las usadas por las empresas en sus predios.

Cuadro 1 - Localización geográfica, especie plantada, momento de instalación y datos químicos de los suelos, en los diferentes sitios experimentales.

Tabela 1 - Localização geográfica, espécies plantadas, tempo de instalação e análise química do solo em diferentes locais experimentais.

\begin{tabular}{|c|c|c|c|c|c|c|c|c|c|c|}
\hline Sitio & Latitud & Longitud & $\mathrm{pH}\left(\mathrm{H}_{2} \mathrm{O}\right)$ & $\mathrm{C}$ & $\mathrm{P}$ & B & $\mathrm{Ca}$ & $\mathrm{Mg}$ & $\mathrm{K}$ & $\mathrm{Na}$ \\
\hline & & & & $\%$ & \multicolumn{2}{|c|}{$\mathrm{mg} \mathrm{kg}^{-1}$} & 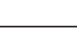 & $-\mathrm{cmc}$ & $\mathrm{kg}^{-1}$ & \\
\hline 1 & $31^{\circ} 16^{\prime} \mathrm{S}$ & $55^{\circ} 43^{\prime} \mathrm{O}$ & 4,45 & 0,99 & 4 & 0,18 & 0,58 & 0,33 & 0,19 & 0,33 \\
\hline 2 & $32^{\circ} 49^{\prime} \mathrm{S}$ & $58^{\circ} 01^{\circ} \mathrm{O}$ & 5,33 & 2,13 & 4 & 0,49 & 7,50 & 1,28 & 0,57 & 0,27 \\
\hline 3 & $34^{\circ} 17^{\prime} \mathrm{S}$ & $54^{\circ} 11^{\prime} \mathrm{O}$ & 5,07 & 1,51 & 4 & 0,65 & 2,00 & 1,00 & 0,48 & 0,33 \\
\hline
\end{tabular}

$\mathrm{pH}\left(\mathrm{H}_{2} \mathrm{O}\right)$ : relación suelo:agua $=1: 2,5$

C: Oxidación con $\mathrm{K}_{2} \mathrm{Cr}_{2} \mathrm{O}_{7} 1 \mathrm{M}+\mathrm{H}_{2} \mathrm{SO}_{4}$ y titulación con $\mathrm{FeSO}_{4}\left(\mathrm{NH}_{4}\right)_{2} \mathrm{SO}_{4} \cdot 6 \mathrm{H}_{2} \mathrm{O}$ (WALKLEY Y BLACK, 1934).

P: Bray-1, $\mathrm{NH}_{4} \mathrm{~F}$ 0,03M y HCl 0,025M (BRAY; KURZT, 1945).

$\mathrm{B}$ : extracción con $\mathrm{BaCl}_{2}\left(1,25 \mathrm{gL}^{-1}\right)$ y determinación colorimética con Azometina H (EMBRAPA, 1999).

$\mathrm{Ca}, \mathrm{Mg}, \mathrm{K}$ y Na: extracción con KCl $1 \mathrm{M}$ a pH 7 y determinación por absorción atómica (Ca, Mg) y emisión (K y Na) (ISAAC; KERBER, 1971).

Cuadro 2 - Clasificación y material de origen de los suelos, en cada sitio experimental.

Tabela 2 - Classificação e material de origem dos solos, em cada local experimental.

\begin{tabular}{cllll}
\hline Sitio & $\begin{array}{l}\text { Unidad de } \\
\text { suelos }^{1}\end{array}$ & Uruguay $^{1}$ & $\begin{array}{l}\text { Clasificación de suelos } \\
\text { Soil Taxonomy }\end{array}$ & Material de origen \\
\hline 1 & Rivera & Acrisol Ócrico & Fine, mixed, semiactive, & Removilizaciones de \\
& & Abrúptico Ar r. & thermic Typic Hapludult & Areniscas de Tacuarembó \\
2 & Tres Bocas & $\begin{array}{l}\text { Argisol Dístrico Ocrico } \\
\text { Abrúptico Ar hm. }\end{array}$ & $\begin{array}{l}\text { Fine, mixed, superactive, } \\
\text { thermic Typic Argiudoll }\end{array}$ & Sedimentos Areno-Arcillosos \\
& & Litosol Subéutrico, & Coarse-loamy, mixed, superactive, & Basamento Cristalino \\
& Polanco & Melánico ArFr. & thermic Lithic Hapludoll & \\
\hline
\end{tabular}

${ }^{1}$ MAP/DSF, 1976.

${ }^{2}$ SOIL SURVEY STAFF, 1999. 
El diseño experimental fue en bloques con parcelas al azar, con cuatro repeticiones. Los tratamientos fueron: testigo (T), solubor (SB), solubor refertilizado (SBR), ulexita (U), ulexita refertilizado (UR). Cada parcela incluía un total de diez árboles distribuidos en dos filas, con un árbol sin fertilizar como borde compartido para separar parcelas en la misma fila, y una fila sin fertilizar como borde compartido hacia los costados. Se evaluó la totalidad de árboles existentes en cada parcela.

Las muestras de suelo, tomadas de $0-20 \mathrm{~cm}$ de profundidad, fueron secadas por 48 horas a $40^{\circ} \mathrm{C}$ y molidas hasta un tamaño menor a $2 \mathrm{~mm}$. En cada muestra se midió pH en agua por potenciometría (relación suelo/ agua $=1 / 2,5)$. El carbono orgánico fue determinado por titulación con sulfato ferroso, luego de atacar una muestra con dicromato de $\mathrm{K}$ y ácido sulfúrico, sin calor exterior (WALKLEY; BLACK, 1934). El P asimilable fue analizado por el método Bray-1 (BRAY; KURZT, 1945). La extracción de Ca, Mg, K y Na se realizó con acetato de amonio $1 \mathrm{M}$ buffereado a $\mathrm{pH}$ 7, determinándose por absorción atómica $(\mathrm{Ca}, \mathrm{Mg})$ y emisión (K y Na) (ISAAC; KERBER, 1971). El B se extrajo por hervido en microondas durante 5 minutos con una solución de $1,25 \mathrm{gL}^{-1}$ de $\mathrm{BaCl}_{2}$, determinándose por el método de Azometina H (EMBRAPA, 1999).

Se realizaron muestreos foliares a $\operatorname{los} 6,12$ y 24 meses de la fertilización (12, 18 y 30 meses del transplante; se mencionarán como $1^{\mathrm{er}}, 2^{\mathrm{do}}$ y $3^{\mathrm{er}}$ muestreo), tomándose la última hoja totalmente desarrollada del último crecimiento, de la mitad de la copa y de los cuatro puntos cardinales. Las muestras fueron secadas a $65^{\circ} \mathrm{C}$ hasta peso constante y molidas. Se analizó el contenido total de B mediante una digestión por vía seca (cenizas), determinándose por el método de Azometina-H (MALAVOLTA et al., 1997).

El análisis estadístico de los datos se realizó mediante Análisis de Varianza (por sitio y para el conjunto de los sitios de igual edad), realizándose contrastes ortogonales entre distintos tratamientos. Se utilizó el programa InfoStat (2008).

\section{RESULTADOS}

En los cuadros 3 y 4 se muestra la concentración foliar de boro y el análisis estadístico, para cada tratamiento, sitio, y para el promedio de los sitios, en los distintos muestreos.
En los dos primeros muestreos, en todos los sitios, las concentraciones foliares de B en los tratamientos fertilizados fueron siempre estadísticamente superiores que las concentraciones de las plantas testigo. Comparando entre fuentes, en los sitios 1 y 3 se observan concentraciones de B en planta más altas en los tratamientos fertilizados con U que en aquellos que recibieron SB. No obstante, estos valores son estadísticamente diferentes solo en el sitio 3 (Cuadro 3).

En el muestreo realizado a los 24 meses post fertilización, si bien se mantiene una diferencia significativa "testigo vs. resto" para la media de los sitios, solo en el sitio 1 existe una diferencia estadística clara $(\mathrm{P}<10)$ (Cuadro 4). En este muestreo no se observan diferencias entre SB y U, pero sí un efecto significativo asociado a la refertilización (contraste 3: "SB+U vs. SBR+UR) para los sitios 1 y 2 , y para el promedio de los sitios. En el sitio 3, las plantas refertilizadas con $\mathrm{U}$ mostraron concentraciones foliares de B significativamente mayores que las refertilizadas con SB $(\mathrm{P}=0,09)$.

\section{DISCUSIÓN}

En primer lugar cabe destacar que no se encontraron concentraciones foliares de $\mathrm{B}$ en el rango considerado como de deficiencia ( $<10 \mathrm{mg} \mathrm{kg}^{-1}$ para E. globulus, $<8 \mathrm{mg} \mathrm{kg}^{-1}$ para E. grandis, árboles de 1-5 años, BOARDMAN et al., 1997) en ninguno de los experimentos, lo que podría deberse a que en el período de estudio no se habrían dado condiciones que favorecieran su aparición.

Las concentraciones foliares de los testigos al $1^{\text {er }}$ muestreo fueron menores que en el $2^{\text {do }}$ y $3^{\text {er }}$ muestreo, para el análisis conjunto y para la mayoría de los sitios. Este aumento podría adjudicarse a una exploración radicular más exhaustiva y/o a una mayor eficiencia de absorción al aumentar la edad de las plantas, la cual les permitiría absorber suficiente $\mathrm{B}$ del suelo para alcanzar niveles de suficiencia.

Las mayores concentraciones foliares de B de los tratamientos fertilizados, indicarían que ambas fuentes presentaron una buena disponibilidad para las plantas en los dos años posteriores a la aplicación. Contrariamente a lo esperado, la fuente U se comportó de manera similar a SB en cuanto a disponibilidad de B para las plantas. Probablemente la acidez de los suelos

Revista Árvore, Viçosa-MG, v.36, n.6, p.1191-1197, 2012 
Cuadro 3 - Concentración foliar de boro $\left(\mathrm{mg} \mathrm{kg}^{-1}\right)$ y análisis estadístico para cada tratamiento y sitio y para el promedio de los sitios, en los dos primeros muestreos. Tratamientos: T: testigo; SB: solubor; U: ulexita.

Tabela 3 - Concentração foliar de boro $\left(\mathrm{mg} \mathrm{kg}^{-1}\right)$ e análise estatística para cada tratamento e local e para a média dos locais, nas duas primeiras amostragens. Tratamentos: T: testemunha, SB: Solubor, U: ulexita.

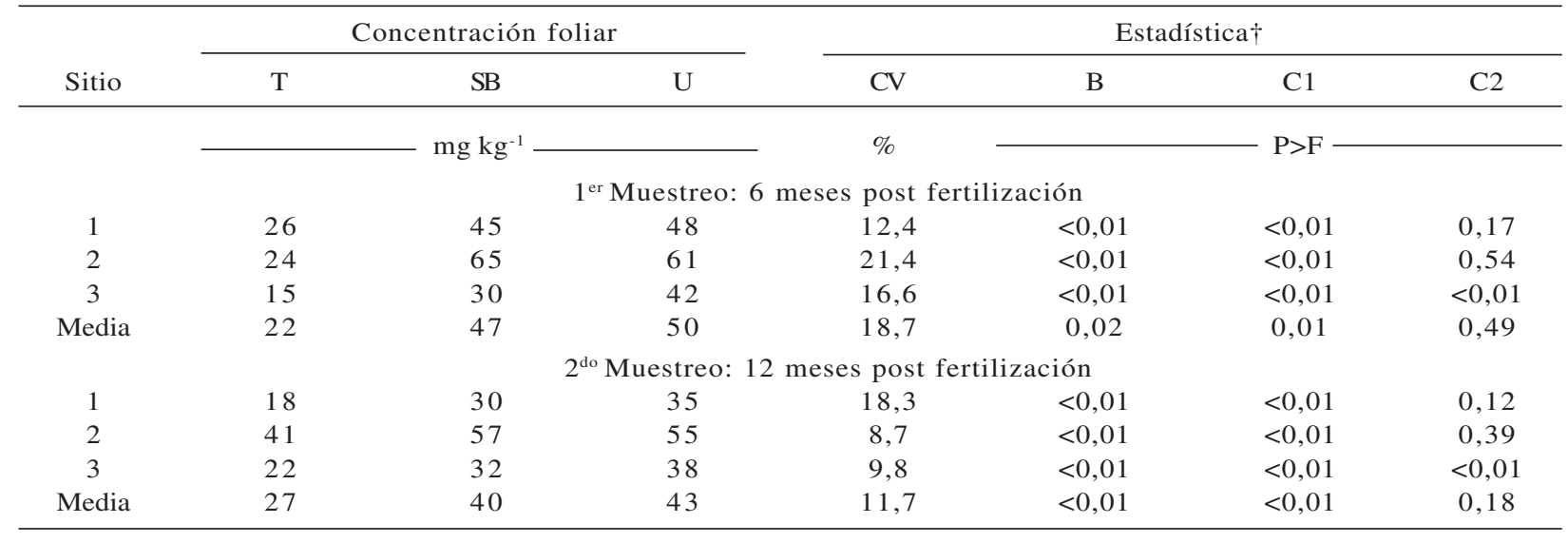

$\dagger$ CV: Coeficiente de Variación; B: boro (efecto principal); C1 y C2 corresponden los contrastes ortogonales "Testigo vs. Resto" y "SB vs. U" respectivamente. Interacción tratamiento*sitio: $1^{\text {er }}$ muestreo $\mathrm{P}<0,01 ; 2^{\text {do }}$ muestreo $\mathrm{P}=0,12$.

Cuadro 4 - Concentración foliar de boro $\left(\mathrm{mg} \mathrm{kg}^{-1}\right)$ y análisis estadístico para cada tratamiento y sitio y para el promedio de los sitios, 24 meses post fertilización y 12 post refertilización. Tratamientos: T: testigo; SB: solubor; SBR: solubor refertilizado; U: ulexita; UR: ulexita refertilizado.

Tabela 4 - Concentração foliar de boro $\left(\mathrm{mg} \mathrm{kg}^{-1}\right)$ e análise estatística para cada tratamento e local e para a média dos locais, 24 meses pós adubação e 12 após adubação de cobertura. Tratamentos: T: testemunha, SB: Solubor, SBR: Solubor em cobertura; U: ulexita; UR: ulexita em cobertura.

\begin{tabular}{|c|c|c|c|c|c|c|c|c|c|c|c|}
\hline \multirow[b]{2}{*}{ Sitio } & \multicolumn{5}{|c|}{ Concentración foliar } & \multicolumn{6}{|c|}{ Estadística $\dagger$} \\
\hline & $\mathrm{T}$ & SB & SBR & $\mathrm{U}$ & UR & $\mathrm{CV}$ & $\mathrm{B}$ & $\mathrm{C} 1$ & $\mathrm{C} 2$ & $\mathrm{C} 3$ & $\mathrm{C} 4$ \\
\hline & & & $\mathrm{ng} \mathrm{kg}$ & & 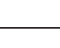 & $\%$ & & & $\mathrm{P}>\mathrm{F}-$ & 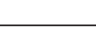 & \\
\hline 1 & 46 & 51 & 63 & 50 & 64 & 17,3 & 0,07 & 0,07 & 0,93 & 0,02 & 0,95 \\
\hline 2 & 51 & 51 & 59 & 52 & 64 & 12,5 & 0,06 & 0,20 & 0,86 & 0,01 & 0,33 \\
\hline 3 & 25 & 29 & 26 & 28 & 31 & 14,1 & 0,22 & 0,10 & 0,74 & 0,91 & 0,09 \\
\hline Media & 41 & 43 & 49 & 43 & 53 & 15,6 & 0,02 & 0,03 & 0,95 & $<0,01$ & 0,30 \\
\hline
\end{tabular}

$\dagger \mathrm{CV}$ : Coeficiente de Variación; B: boro (efecto principal); C1, C2, C3 y C4 corresponden a los contrastes ortogonales "Testigo vs. Resto", "SB vs. U", "SB+U vs. SBR+UR", y "SBR vs. UR" respectivamente. Interacción tratamiento*sitio: P = 0,34.

$\left(\mathrm{pH}_{\mathrm{H} 2 \mathrm{O}}\right.$ entre 5,33 y 4,45) influyó de manera importante en la solubilización de la fuente menos soluble. El comportamiento de U, similar a una fuente soluble, se evidenció en los aumentos de las concentraciones foliares en todos los experimentos, aún siendo la aplicación realizada en cobertura. Posiblemente la existencia de diferente granulometría dentro del fertilizante hizo que existiera diferente solubilidad. Según Shorrocks (1997), las partículas finas de ulexita se comportan de manera similar al bórax (tanto incorporada al suelo como aplicada en superficie), mientras que las partículas grandes de ulexita proporcionarían B por más tiempo.
En el sitio 3, forestado con E. globulus, las concentraciones foliares de los tratamientos fertilizados con U fueron significativamente superiores a los fertilizados con SB, lo cual podría estar indicando un mejor aprovechamiento del B proveniente de esta fuente por parte de esta especie. Las diferencias no parecerían estar asociadas a características del suelo o clima, aunque no deberían descartarse.

Para el promedio de los sitios, se observa una gradual pero permanente caída en las concentraciones foliares de los tratamientos fertilizados. Al año del 
transplante, si bien disminuyeron las concentraciones foliares, todavía se observó residualidad de la fertilización. Luego de 24 meses de la aplicación, las concentraciones foliares de los tratamientos fertilizados disminuyeron hasta valores que, aunque fueron levemente superiores, no se diferenciaron significativamente de los testigos, manteniéndose igualmente dentro del rango de suficiencia.

Hunter et al. (1990), trabajando en plantaciones de pino, encontraron que las concentraciones foliares de plantaciones fertilizadas con boratos de sodio mostraron un pico de concentración al año de la aplicación, con posterior caída en los sucesivos años. Las ulexitas molidas gruesas (2-5 mm), por el contrario, mostraron una residualidad de hasta al menos cuatro años post aplicación. En nuestro trabajo, la residualidad de $U$, evaluada a través de las concentraciones foliares, sería similar a SB, no existiendo diferencias significativas entre los valores determinados en muestras foliares tomadas a los 24 meses post fertilización, en los tratamientos fertilizados con ambas fuentes.

La dosis de B aplicada (4 g de B por planta) parecería ser suficiente para alcanzar niveles foliares que podrían considerarse de suficiencia (de 12 a $50 \mathrm{mg} \mathrm{kg}^{-1}$ y de 15 a $30 \mathrm{mg} \mathrm{kg}^{-1}$ para E. globulus, y E. grandis, respectivamente, para árboles de 1-5 años, según BOARDMAN et al., 1997), sin llegar a niveles de toxicidad.

\section{CONCLUSIÓN}

Los resultados de este estudio permiten afirmar que en los suelos estudiados, los fertilizantes del tipo ulexita utilizados en este experimento, tendrían similar eficiencia que los boratos de sodio, como aporte de B para las plantaciones de eucaliptos. Esta ulexita, a pesar de ser un borato de sodio y calcio, se comportó como de alta solubilidad y de media a baja residualidad, presentado un comportamiento similar al del borato de sodio.

\section{AGRADECIMIENTOS}

A las empresas Colonvade S.A., Eufores S.A., Forestal Oriental S.A., Grupo Forestal Chileno S.A., así como a la Comisión Sectorial de Investigación Científica de la Universidad de la República, por el apoyo financiero.

\section{REFERENCIAS}

BOARDMAN, R. et al. Forest plantations. In: REUTER, D. J.; ROBINSON, J. B. (Ed.). Plant analysis, an interpretation manual. 2.ed. Collingwood: CSIRO, 1997. p.505-561.

BRAY, R. H.; KURTZ, L. T. Determination of total, organic and available forms of phosphorus in soils. Soil Science, v.59, n.1, p.39-45, 1945.

BROWN, P. H.; SHELP, B. J. Boron mobility in plants. Plant and Soil, v.193, n.1, p.85-101, 1997.

COOLING, E. N.; JONES, B. E. The importance of boron and NPK fertilizers to Eucalyptus in the Southern Province, Zambia. East African Agricultural and Forestry Journal, v.36, n.2, p.185-194, 1970.

DELL, B. Diagnosis of nutrient deficiencies in Eucalypts. In: ATTIWIILL, P.M.; ADAMS, M.A. (Ed). Nutrition of Eucalypts. Melbourne: CSIRO, 1996. p.417-440.

DELL, B.; HUANG, L. Physiological response of plants to low boron. Plant and Soil, v.193, n.1/2, p.103-120, 1997.

DELL, B.; MALAJCZUK, N. Boron deficiency in eucalypt plantations in China. Canadian Journal Foresty of Research, v.24, n.12, p.2409-2416, 1994.

DUGGER, W. M. Boron in plant metabolism. In: LAÜCHI, A.; BIELESKI, R. L. (Ed).

Encyclopedia of plant physiology. Berlín: Springer-Verlag, 1983. p.626-650. (New Series, 15b).

EMPRESA BRASILEIRA DE PESQUISA AGROPECUÁRIA - EMBRAPA. Manual de análises químicas de solos, plantas e fertilizantes. Brasília: Embrapa Solos/Embrapa Informática Agropecuária/Embrapa Comunicação para Transferência de Tecnologia, 1999. 370p.

HAVLIN, J. L. et al. Soil fertility and fertilizers: an introduction to nutrient management. 7.ed. Pearson: Upper Saddle River, 2005. 515p.

HOPMANS, P.; FLINN, D. W. Boron deficiency in Pinus radiata D. Don and the effect of applied boron on height growth and nutrient uptake. Plant and Soil, v.79, n.2, p.295-298. 1984. 
HUNTER, I. R.; WILL, G. M.; SKINNER, M. F. A strategy for correction of boron deficiency in radiata pine plantations in New Zealand. Forest Ecology and Management, v.37, n.1/3, p.77-82, 1990.

INFOSTAT. Grupo InfoStat. Córdoba: FCA, Universidad Nacional de Córdoba, 2008.

ISAAC, R. A.; KERBER, J. D. Atomic Absorption and flame photometry: techniques and uses in soil, plant and water analysis. In: WALSH, L. M. (Ed.). Instrumental methods for analysis of soil and plant tissues. Madison: Soil Science Society of America, 1971. p.17-37.

LEITE, S. M. M.; MARINO, C. L.; BONINE,C. A. V. Respostas de clones de Eucalyptus grandis e E. grandis x E. urophylla à supressão de boro. Scientia Forestalis, v.38, p.19-25, 2010.

LEITE, S. M. M. et al. Influence on concentration of polyols and other sugars in Eucalyptus.

Revista Árvore, v.32, n.5, p. 815-820, 2008.

MALAVOLTA, E.; VITTI, G. C.; OLIVEIRA, S. A. Avaliação do estado nutricional das plantas: princípios e aplicações. 2.ed. Piracicaba: Associação Brasileira para Pesquisa da Potassa e do Fosfato, 1997. 319p.

URUGUAY. Ministerio de Agricultura y Pesca, Dirección de Suelos y Fertilizantes MAP/DSF. Carta de Reconocimiento de Suelos del Uruguay. Montevideo: 1976.

MATTIELLO, E. M. et al. Características fisiológicas e crescimento de clones de eucalipto em resposta ao boro. Revista Árvore, v.33, n.5, p.821-830, 2009.

MATOH, T. Boron in plant cell walls. Plant and Soil, v.193, n.1/2, p.59-70, 1997.

MÉNDEZ, A. Evaluación del estado nutricional en plantaciones de $E$. globulus Labill. ssp. globulus. 2003. 95f. Tesis (Ing. Agr ) - Faculdad de Agronomía, Montevideo, Uruguay, 2003.

MENGEL, K.; KIRKBY, E. A. Principles of plant nutrition. 5.ed. Dordrecht: Kluwer, 2001.849p.
REALI, P. Daños por falta de boro. Disminución de la rentabilidad forestal en E. globulus ssp. globulus en el Sureste del Uruguay. Forestal, v.15, n.1, p.25-27, 2000.

SAVORY, B. M. Boron deficiency in Eucalyptus in Northern Rhodesia. Empire Forestry Review, v.41, n.2, p.118-26, 1962.

SAYAGUÉS, L.; MÉNDEZ, A. Deficiencia de boro en E. globulus ssp. globulus en Uruguay. Forestal, v.30, n.1, p.15-18, 2006.

SHORROCKS, V. M. The occurrence and correction of boron deficiency. Plant and Soil, v.193, n.1/2, p.121-148, 1997.

SILVEIRA, R. L. V. A.; GONÇALVES, A. N.; KRÜGNER, T. L. Estado nutricional de Eucalyptus citriodora Hook cultivado sob diferentes doses de boro e sua relação com a agressividade de Botryosphaeria ribis. Scientia Forestalis, v.53, n.1, p.57-70. 1998.

SILVEIRA, R. L. V. A. et al. F. Sintomas de deficiência de macronutrientes e de boro em clones híbridos de Eucalyptus grandis com Eucalyptus urophylla. Cerne, v. 8, n.2, p. 108-117, 2002.

SOIL SURVEY STAFF. Soil taxonomy: a basic system of soil classification for making and interpreting soil surveys. 2.ed. Washington, DC: NRCS, 1999. 436p.

STONE, E. L.; WILL, G. M. Boron deficiency in Pinus radiate and Pinus pinaster. Forest Science, v.11, n.4, p.425-433, 1965.

TOKESHI, H.; GUIMARÃES, R. F.; TOMAZELLO FILHO, M. Deficiência de boro em Eucalyptus em São Paulo. Summa Phytopathologica, v.2, n.1/2, p.122-126, 1976.

VAIL, J. W.; PARRY, M. S.; CALTON, W. E. Boron deficiency dieback in pines. Plant and Soil, v.14, n.4, p.393-398. 1961.

WALKLEY, A.; BLACK, T. A. An examination of the degtjareff method for determining soil organic matter and a proposed modification of the chromic acid titration method. Soil Science, v.37, n.1, p.29-38, 1934.

Revista Árvore, Viçosa-MG, v.36, n.6, p.1191-1197, 2012 
